\section{Response to Letter to the Editor: Isolated Tumor Cells in Sentinel Lymph Nodes and Clinical Implications for Early Breast Cancer}

\section{TO THE EDITORS:}

We appreciate the letter to the editor from Dr. Ziogas and colleagues written in response to our work investigating clinicopathologic factors associated with identifying isolated tumor cells (ITC) in sentinel lymph nodes (SLN) from early-stage breast cancer patients. We were particularly interested in their comments regarding personalized medicine as we would contend that sentinel lymph node dissection (SLND) represents personalized surgery. SLND allows surgeons to selectively perform complete axillary lymph node dissections only on those patients whose SLNs have proven metastatic disease. These patients may benefit from both improved staging and ensuring regional control. Unfortunately, as reiterated in this letter to the editor, it remains unknown as to how we should treat patients with small-volume disease, including ITCs (metastasis $\leq 0.2 \mathrm{~mm}$ in size) and micrometastases ( $>0.2$ to $2.0 \mathrm{~mm}$ in size), identified in their SLNs.

In our article, we identified that lobular histology and presence of lymphovascular invasion (LVI) are associated with increased incidence of ITCs in SLNs. ${ }^{1}$ Importantly, these findings suggest that the biology of the tumor may be important in distinguishing subsets of patients with these small-volume metastases. Specifically, metastases from invasive lobular carcinoma frequently present as small foci scattered throughout the lymph node that may only be detected by pathologic examination as ITCs. LVI has clearly been demonstrated to be a poor prognostic factor in breast cancer. The relationship between LVI and presence of ITCs suggests that it is reasonable to consider performing completion axillary lymph node dissections in patients with ITCs in their SLNs if the primary tumor also has LVI.

Personalized medicine with respect to cancer care implies treating individual patients based on the biology of their disease. We, like others, await the long-term followup of the American College of Surgeons Oncology Group Z0010 trial and the National Surgical Adjuvant Breast and Bowel Project B-32 trial results to provide more information regarding the clinical relevance of these small-volume metastatic deposits in lymph nodes. Until the time that the cooperative group trial data mature, we contend that our findings that specific biologic factors are associated with finding ITCs in the sentinel lymph nodes represent an advancement in personalizing the surgical care of earlystage breast cancer patients.

Elizabeth A. Mittendorf, MD, and Kelly K. Hunt, MD Department of Surgical Oncology, The University of Texas M. D. Anderson Cancer Center, Houston, TX e-mail: khunt@mdanderson.org

Published Online: 9 April 2009

(C) Society of Surgical Oncology 2009

\section{REFERENCE}

1. Mittendorf EA, Sahin A, Tucker SL, et al. Lymphovascular invasion and lobular histology are associated with increased incidence of isolated tumor cells in sentinel lymph nodes from early-stage breast cancer patients. Ann Surg Oncol. 2008;15:3369-77. 